\title{
SUPPORT POINTS OF THE SET OF UNIVALENT FUNCTIONS
}

\author{
LOUIS BRICKMAN ${ }^{1}$ AND DONALD WILKEN ${ }^{2}$
}

\begin{abstract}
Let $S$ be the usual set of analytic, univalent, normalized functions on the unit disk $\Delta$. Let $f \in S$. Then $f$ is $a$ support point of $S$, if there exists a continuous linear functional $J$ on the space of analytic functions on $\Delta, J$ nonconstant on $S$, such that $\operatorname{Re} J(f)=\max \{\operatorname{Re} J(g): g \in S\}$. TheOREM. Let $f$ be a support point of $S$. Then $f$ is analytic in the closed unit disk except for a pole of order two at one point of the unit circle. The complement of $f(\Delta)$ is a single arc $\Gamma$, regular and analytic everywhere, tending to $\infty$ in such $a$ way that the angle between $\Gamma$ and the radial direction is always less than $\pi / 4$. Near $\infty, \Gamma$ can be described in the form $\sum_{n=-1}^{\infty} d_{n} t^{n}$ $\left(0<t<\delta, d_{-1} \neq 0\right)$. In particular, $\Gamma$ is asymptotic to a line $d_{-1} t^{-1}+d_{0}$.
\end{abstract}

Let $\Delta$ be the open unit disk of the complex plane $C$, and let $J$ be a continuous linear functional on the space of functions analytic on $\Delta$. (The continuity of $J$ means that if $g_{n} \rightarrow g$ uniformly on compact subsets of $\Delta$, then $J\left(g_{n}\right) \rightarrow J(g)$ in $C$.) We assume further that $J$ does not have the "trivial" form $J(g)=\alpha g(0)+\beta g^{\prime}(0)$. Let $S$ be the usual set of analytic univalent functions $g$ on $\Delta$ satisfying $g(0)=0$ and $g^{\prime}(0)=1$. If $f \in S$ we shall say that $f$ is a support point of $S$ provided there exists a functional $J$ as described above such that

$$
\operatorname{Re} J(f)=\max \{\operatorname{Re} J(g): g \in S\} .
$$

In geometrical language $S$ has a "nontrivial" supporting hyperplane passing through $f$.

We shall establish the following theorem.

THEOREM. Let $f$ be a support point of $S$. Then the following statements hold.

I. $f$ is analytic in the closed unit disk $\bar{\Delta}$ except for a pole of order two at one point of the unit circle $\partial \Delta$.

Received by the editors January 29, 1973 and, in revised form, May 21, 1973.

AMS (MOS) subject classifications (1970). Primary 30A36, 30A38; Secondary $30 \mathrm{~A} 40$.

${ }^{1}$ Research partially supported by National Science Foundation Grant PO 19709000.

${ }^{2}$ Research partially supported by National Science Foundation Grant PO 12020000 , and a State University of New York Fellowship. 
II. $C \backslash f(\Delta)$ is a single arc $\Gamma$, regular and analytic everywhere (including the endpoint), tending to $\infty$ with increasing modulus. In fact the angle between $\Gamma$ and the radius vector to any point of $\Gamma$ is less than $\pi / 4$. (We have not established strict inequality at the endpoint of $\Gamma$.)

III. Near $\infty, \Gamma$ can be described in the form $\sum_{n=-1}^{\infty} d_{n} t^{n}(0<t<\delta$, $\left.d_{-1} \neq 0\right)$. In other words, $\Gamma$ is regular and analytic at $\infty$. In particular $\Gamma$ is asymptotic to a line $d_{-1} t^{-1}+d_{0}$.

Our principal tool will be the result of Schiffer (see [6], [7], and [9, p. 401]) that $C \backslash f(\Delta)$ consists of (countably many, regular) analytic arcs, each admitting a parameterization satisfying the differential equation

$$
J\left(f^{2} /(f-w)\right)(d w / d t)^{2}=w^{2} .
$$

(In particular we note that a bounded function cannot be a support point of $S$.) This result is an easy consequence of Schiffer's lemma on boundary variations [6] if one first shows that $J\left(f^{2} /(f-w)\right)$ is an analytic function of $w$, not identically zero, in a suitable region containing $C \backslash f(\Delta)$. We prove the analyticity in Lemma 1 but we omit the (easy) proof that $J\left(f^{2} /(f-w)\right) \equiv 0$ implies that $J$ has the trivial form described above. We shall also make use of the construction in [1]. Since these tools are not restricted to the unit disk, appropriate modifications of our statements II and III hold for more general regions. We expect (but have not proved) the following result if $\Delta$ is replaced by a region $\Omega$ containing 0 : Each arc contained in $C \backslash f(\Omega)$ is regular and analytic everywhere and has the $\pi / 4$ property of II, exactly one such arc is unbounded, and this arc has the behavior at $\infty$ described in III.

Some well-known results that overlap our theorem are as follows. In $\left[2\right.$, p. 147] it is proven that if $J$ has the special form $J(g)=\sum_{v=1}^{n} b_{v} g^{(v)}(0)$, $n \geqq 2$, then $C \backslash(f \Delta)$ consists of finitely many analytic arcs with the $\pi / 4$ property. In [5] it is shown that if $J(g)=g^{(n)}(0) / n !, n \geqq 2$, then $C \backslash f(\Delta)$ consists of a single analytic arc asymptotic at $\infty$ to a line. In [8] (in the context of a region $\Omega$ containing 0$)$ Schiffer shows that if $J(g)=g^{(n)}(0) / n$ !, $n \geqq 2$, then $J\left(f^{2}\right) \neq 0$, and the unbounded component of $C \backslash f(\Omega)$ has an asymptotic direction at $\infty$. The only previous investigation of support points per se is the important paper of A. Pfluger [4]. Among the many results obtained is the following. If $f$ is a support point of $S$ (not Pfluger's terminology), then $C \backslash f(\Delta)$ is a single analytic arc with the $\pi / 4$ property (as described in II above but apparently not with strict inequality). The proof given in [4] is considerably more difficult than our proof of II.

We shall divide the proof of our theorem into several lemmas. Throughout the proof $f$ will denote a support point of $S$ with respect to the functional $J$. 
LEMMA 1. $J\left(f^{2} /(f-w)\right)$ is an analytic function of $w$ in the complement of a compact subset of $f(\Delta)$.

Proof. This is an immediate consequence of the following (known) representation theorem for $J$ : There exists a compact set $K \subset \Delta$ and a complex measure $\mu$ such that $J(g)=\int_{K} g d \mu$ for every $g$ analytic on $\Delta$. The proof of this can be outlined as follows. Let $\left\{\Delta_{k}\right\}$ be a sequence of proper, concentric subdisks of $\Delta$ with $\bigcup_{k} \Delta_{k}=\Delta$. Let

$$
\|g\|_{k}=\max \left\{|g(z)|: z \in \bar{\Delta}_{k}\right\} \text {. }
$$

Then for some $k,\left\{|J(g)| /\|g\|_{k}\right\}$ is bounded as $g$ varies over the nonzero analytic functions on $\Delta$. By the Hahn-Banach theorem $J$ extends to a bounded linear functional on the Banach space of continuous functions on $\bar{\Delta}_{k}$. The Riesz representation theorem then gives the required measure $\mu$.

The above proof generalizes easily to an arbitrary region. Specifically for $\Delta$, however, a more explicit result holds: There exists $r, 0<r<1$, and $F$ analytic on $\{z:|z| \geqq r\} \cup\{\infty\}$ such that

$$
J(g)=(1 / 2 \pi) \int_{0}^{2 \pi} g\left(r e^{i \theta}\right) F\left(r e^{i \theta}\right) d \theta
$$

for every $g$ analytic on $\Delta$. In other words, $J(g)=\sum_{n} g_{n} F_{n}$, where

$$
g(z)=\sum_{n} g_{n} z^{n} \text { and } \limsup _{n \rightarrow \infty}\left|F_{n}\right|^{1 / n}<1 .
$$

LeMma 2. $C \backslash f(\Delta)$ consists of a single arc $\Gamma$ tending to $\infty$ with strictly increasing modulus.

Proof. It is sufficient to show that $C \backslash f(\Delta)$ does not contain two points of equal modulus. (See [1].) Suppose this is false. Then, as shown in [1], $f$ is not an extreme point of $S$. That is, there exist $f_{1}, f_{2} \in S\left(f_{1} \neq f_{2}\right)$ and $t \in(0,1)$ such that $f=t f_{1}+(1-t) f_{2}$. But the proof in [1] shows that either $f_{1}$ or $f_{2}$ is bounded, and a bounded function cannot be a support point of $S$. Therefore we reach a contradiction by applying $\operatorname{Re} J$ to the last equation.

Lemma 3. Let $w_{0}$ be the endpoint of $\Gamma$ and let $w \in \Gamma \backslash\left\{w_{0}\right\}$. Then $\operatorname{Re} J\left(f^{2} /(f-w)\right)>0$.

Proof. Let $f_{w}=w f /(w-f)$. Then $f_{w} \in S$ and $C \backslash f_{w}(\Delta)$ consists of two disjoint unbounded arcs. By Lemma 2 (applied to $f_{w}$ ) $f_{w}$ is not a support point of $S$. Therefore $\operatorname{Re} J\left(f_{w}\right)<\operatorname{Re} J(f)$. But $f-f_{w}=f^{2} /(f-w)$, so the desired inequality follows. 
COROLlARY 1. $\Gamma$ is regular and analytic everywhere except possibly at $w_{0}$ and at $\infty$.

Proof. The proof of Schiffer's lemma [6] shows that $\Gamma$ is regular and analytic at each point $w$ such that $J\left(f^{2} /(f-w)\right) \neq 0$.

COROLlaRY 2. $\Gamma$ has the $\pi / 4$ property of statement II.

Proof. Lemma 3 and equation (1) yield $\operatorname{Re}\left[w^{\prime}(t) / w(t)\right]^{2}>0$.

We observe that $J\left(f^{2} /\left(f-w_{0}\right)\right)$ can equal 0 . For example if $J$ is the 3 rd coefficient functional and $f(z)=z /(1-z)^{2}$ (see [3]), then $w_{0}=-\frac{1}{4}$ and $f_{w_{0}}(z)=z /(1+z)^{2}$. Hence $J\left(f-f_{w_{0}}\right)=0$.

LEMMA 4. $\Gamma$ is analytic and regular at $\infty$ as described in III.

Proof. Let $\Gamma$ have the parameterization $w(t)$ satisfying (1). We choose an analytic square root function in a neighborhood of $\Gamma$ and define $v(t)=w(t)^{-1 / 2}$. Then (1) transforms into

$$
J\left(f^{2} /\left(v^{2} f-1\right)\right)(d v / d t)^{2}=\frac{1}{4} .
$$

From the measure theoretic representation of $J$ discussed in Lemma 1, it is clear that $J\left(f^{2} /\left(v^{2} f-1\right)\right)$ is an analytic, even function of $v$ in some neighborhood of 0 . Hence we can extract square roots appropriately in (2) to obtain an equation of the form

$$
v^{m}\left(a_{0}+a_{2} v^{2}+\cdots\right) d v / d t=1 \quad\left(a_{0} \neq 0\right),
$$

where $m$ is a nonnegative integer and $|v(t)|$ is sufficiently small. (Professor Schiffer has kindly informed us that his result $J\left(f^{2}\right) \neq 0$ of [7], where $J$ is an $n$th coefficient functional, will generalize to the present context. Moreover a proof for general $J$ subsequently appeared in [4]. Assuming this, one obtains $m=0$, and the reasoning below becomes simpler. We have preferred, however, to keep the paper independent of this comparatively difficult result.) If we assume that $w(t) \rightarrow \infty(v(t) \rightarrow 0)$ as $t \rightarrow 0^{+}$, integration of the last equation leads to

$$
\frac{a_{0}}{m+1} v^{m+1}+\frac{a_{2}}{m+3} v^{m+3}+\cdots=t \quad\left(a_{0} \neq 0\right)
$$

for $t$ sufficiently small and positive. We then obtain successively

$$
\begin{aligned}
& \left(b_{1} v+b_{3} v^{3}+\cdots\right)^{m+1}=t \quad\left(b_{1} \neq 0\right), \\
& v=c_{1} t^{1 /(m+1)}+c_{3} t^{3 /(m+1)}+\cdots \quad\left(c_{1} \neq 0\right), \\
& w=d_{-2} t^{-2 /(m+1)}+d_{0}+d_{2} t^{2 /(m+1)}+\cdots \quad\left(d_{-2} \neq 0\right) .
\end{aligned}
$$

If we now replace $t^{2 /(m+1)}$ by $t$, and $d_{2 n}$ by $d_{n}(n \geqq-1)$, we obtain III. 
We remark that if $m=0$ (which indeed must be the case) a calculation gives $d_{-1}=-4 J\left(f^{2}\right)$ and $d_{0}=J\left(f^{3}\right) / 3 J\left(f^{2}\right)$.

LEMMA 5. $\Gamma$ is analytic and regular at its endpoint $w_{0}$.

Proof. If $J\left(f^{2} /\left(f-w_{0}\right)\right) \neq 0$, the function $w(t)$ satisfying (1) is the required parameterization. Otherwise $f_{w_{0}}=w_{0} f /\left(w_{0}-f\right)$ is a support point of $S$. (See Lemma 3.) Let $p$ be the parameterization of $C \backslash f_{w_{0}}(\Delta)$ near $\infty$ assured by Lemma 4, and let $l(w)=w_{0} w /\left(w_{0}-w\right)$. Then $l^{-1} \circ p$ is as required.

We remark that $J\left(f^{2} /\left(f-w_{0}\right)\right)=0$ actually implies that $\Gamma$ lies on a straight line through the origin and this in turn implies that $f$ is a Koebe function. Indeed the proof of this, given by Schiffer for $J=n$th coefficient (see [9, pp. 403-405]), is valid for any continuous linear $J$. (See also [4, pp. 24-25].)

Our final lemma is an elaboration of statement I of the theorem.

LEMMA 6. There are two points $z_{0}$ and $z_{1}$ on the unit circle $\partial \Delta$ such that

(i) $f$ is analytic on $\bar{\Delta}$ except for a pole of order 2 at $z_{1}$,

(ii) $f-w_{0}$ has a zero of order 2 at $z_{0}$,

(iii) $f^{\prime}$ is nonvanishing on each of the two open arcs $\left(z_{0}, z_{1}\right)$, and $f$ maps each closed arc $\left[z_{0}, z_{1}\right]$ univalently onto $\Gamma \cup\{\infty\}$.

Proof. By means of three elementary conformal maps and Carathéodory's theorem [2, p. 44] on boundary correspondence of Jordan domains under conformal mappings, we conclude that $f$ has a continuous extension to a map from $\bar{\Delta}$ into the Riemann sphere $C \cup\{\infty\}$, that $f^{-1}\left(w_{0}\right)$ and $f^{-1}(\infty)$ are single points $z_{0}$ and $z_{1}$, and that $f$ maps each arc $\left[z_{0}, z_{1}\right]$ univalently onto $\Gamma \cup\{\infty\}$. But we have shown (Lemma 2, Corollary 1, Lemma 4, Lemma 5) that $\Gamma \cup\{\infty\}$ is a regular, Jordan analytic arc. Therefore we can continue $f$ through $\partial \Delta$ by routine reflection arguments. In doing so we note that the extended function is locally two-to-one at $z_{0}$ and $z_{1}$, and locally univalent elsewhere on $\partial \Delta$. Thus our lemma follows and the proof of the theorem is complete.

The authors would like to thank Professor Richard Hornblower for several stimulating conversations.

\section{BIBLIOGRAPHY}

1. L. Brickman, Extreme points of the set of univalent functions, Bull. Amer. Math. Soc. 76 (1970), 372-374. MR 41 \#448.

2. G. M. Goluzin, Geometric theory of functions of a complex variable, GITTL, Moscow, 1952; English transl., Transl. Math. Monographs, vol. 26, Amer. Math. Soc., Providence, R.I., 1969. MR 15, 112; 40 \#308.

3. K. Löwner, Untersuchungen über schlichte konforme Abbildungen des Einheitkreises. I, Math. Ann. 89 (1923), 103-121. 
4. A. Pfluger, Lineare Extremalprobleme bei schlichten Funktionen, Ann. Acad. Sci. Fenn. Ser. AI no. 489 (1971), 32 pp. MR 45 \#5337.

5. A. C. Schaeffer and D. C. Spencer, Coefficient regions for schlicht functions, Amer. Math. Soc. Colloq. Publ., vol. 35, Amer. Math. Soc., Providence, R.I., 1950. MR 12, 326.

6. M. Schiffer, A method of variation within the family of simple functions, Proc. London Math. Soc. (2) 44 (1938), 432-449.

7. - Extremum problems and variational methods in conformal mapping, Proc. Internat. Congress Math., 1958, Cambridge Univ. Press, New York, 1960, pp. 211-231. MR 24 \#A3287.

8. - On the coefficient problem for univalent functions, Trans. Amer. Math. Soc. 134 (1968), 95-101. MR 37 \#4249.

9. M. Schiffer and D. C. Spencer, Functionals of finite Riemann surfaces, Princeton Univ. Press, Princeton, N.J., 1954. MR 16, 461.

Department of Mathematics, State University of New York at Albany, Albany, New York 12222 